\title{
The physical structure of high-mass star-forming cores
}

\author{
J. Hatchell and F. F. S. van der Tak
}

\author{
Max-Planck-Institut für Radioastronomie, Auf dem Hügel 69, 53121 Bonn, Germany \\ e-mail: hatchell@mpifr-bonn.mpg.de, vdtak@mpifr-bonn.mpg.de
}

Received 4 April 2003 / Accepted 11 July 2003

\begin{abstract}
We present models of the temperature and density structure of the envelopes of ten deeply embedded massive stars associated with ultracompact HII regions. Constraints come from 60-1300 $\mu \mathrm{m}$ photometry including ISO-LWS spectra, maps at 450 and $850 \mu \mathrm{m}$, and CS emission line data. Radial profiles extracted from the maps after removing neighbouring sources were modelled taking the chopping process into account. The line data are modelled with a Monte Carlo program and the continuum data with a dust radiative transfer code. For an assumed $n(r) \propto r^{-p}$ density structure, the index $p$ is found to be uniformly distributed between 1.25 and 2.25 for this sample. The density power law index from radial profiles and emission lines agree well (by \pm 0.25 ), while the continuum spectrum sometimes deviates (by \pm 0.5 ). Reliable models thus require all three kinds of data.
\end{abstract}

Key words. stars: formation - ISM: dust, extinction - ISM: HII regions - ISM: molecules - ISM: structure

\section{Introduction}

The advent of bolometer arrays and on-the-fly mapping techniques on single-dish (sub-)millimeter telescopes has greatly improved our knowledge of the structure of embedded young stellar objects (see André et al. 2000 for a review). Recently, Shirley et al. (2002) and Jørgensen et al. (2002) developed detailed models for low-mass envelopes.

Of particular interest are the envelopes of recently formed high-mass stars. The formation of high-mass stars may be a scaled-up version of the process for low-mass stars, which are known to form by accretion via a disk, and to disperse their envelopes through bipolar outflows. Alternatively, high-mass stars may form by the coagulation of lower mass stars or of protostellar cores. Determining the structure of the envelopes of recently formed high-mass stars may enable us to decide between the two scenarios. Models of the large-scale structure of the envelopes is also a prerequisite for measuring the sizes and masses of any circumstellar cores or disks. Third, the temperature and density structure is essential input for models of the chemistry of these regions, which often have rich molecular line spectra. Not only are they important as testbeds for theories of interstellar chemistry, but the chemical composition of star-forming matter is expected to change with time, which would allow one to determine ages through molecular abundances. An example is the "hot core" phenomenon which is likely to be short-lived. However, its exact evolutionary status and the link with any physical component have not yet been firmly established.

Send offprint requests to: J. Hatchell, e-mail: hatchell@mpifr-bonn.mpg.de
The physical structure of regions of high-mass star formation has been investigated by Hatchell et al. (2000), van der Tak et al. (2000), Beuther et al. (2002) and Mueller et al. (2002). These studies indicated power-law density distributions, possibly with central cores. Power law indices varied from 1.0 to 2.0, which would make high-mass envelopes scaled-up versions of the envelopes of young low-mass stars. However, this similarity is not proof that the dynamics of both cases are similar, since the spatial scales differ by an order of magnitude. In addition, while Hatchell et al. found a central steepening of the density profile, Beuther et al. found a central flattening. The discrepancy may be due to non-overlapping source samples, but a review of the old results seems in order.

A second motivation to return to the problem is improved information. High quality (SCUBA) submillimeter continuum images are now available for a larger source sample (Thompson et al. 2002), and far-infrared spectra from ISO are now public. Finally, the models of Hatchell et al. fixed $r^{-1.5}$ density laws for the envelopes, while those of van der Tak et al. kept luminosity and outer radius fixed. Recent improvement in computing power allows a more systematic exploration of parameter space and to include the effects of telescope chopping.

Table 1 introduces the source sample for this paper, which is rather small as we limited it to sources for which CS and preferably also ISO data were available. Performing a detailed analysis on a large sample such as that of Beuther et al. is still beyond reach. When drawing conclusions, we assign a lower weight to G12.21 because its distance is much larger than that of the other sources, and its envelope is hardly resolved with SCUBA. The mean distance of the sample, excluding G12.21, is $5.3 \mathrm{kpc}$. 
Table 1. Source sample.

\begin{tabular}{|c|c|c|c|c|c|c|c|}
\hline \multirow[t]{2}{*}{ Object } & \multicolumn{2}{|c|}{ RA (1950) } & \multicolumn{3}{|c|}{ Dec (1950) } & \multirow{2}{*}{$\begin{array}{c}d^{1} \\
\mathrm{kpc}\end{array}$} & \multirow{2}{*}{$\begin{array}{c}\log L^{2} \\
L_{\odot}\end{array}$} \\
\hline & {$\left[\begin{array}{ll}\mathrm{h} & \mathrm{m}\end{array}\right.$} & i s ] & [ deg &, & "] & & \\
\hline G5.89-0.39 & 175 & 726.8 & -24 & 03 & 56 & 2.0 & 4.40 \\
\hline G9.62+0.19 & 180 & 316.2 & -20 & 32 & 03 & 5.7 & 5.60 \\
\hline $\mathrm{G} 10.47+0.03$ & 180 & 540.3 & -19 & 52 & 21 & 5.8 & 5.60 \\
\hline G12.21-0.10 & 180 & 943.7 & -18 & 25 & 09 & 13.5 & 5.95 \\
\hline $\mathrm{G} 13.87+0.28$ & 181 & 141.8 & -16 & 46 & 40 & 4.4 & 5.23 \\
\hline G29.96-0.02 & 184 & 327.1 & -02 & 42 & 36 & 6.0 & 5.90 \\
\hline $\mathrm{G} 31.41+0.31$ & 184 & 459.4 & -01 & 16 & 04 & 7.9 & 5.23 \\
\hline $\mathrm{G} 34.26+0.15$ & 185 & 046.1 & +01 & 11 & 12 & 3.3 & 5.50 \\
\hline $\mathrm{G} 45.47+0.05$ & 1912 & 204.4 & +11 & 04 & 11 & 8.3 & 6.30 \\
\hline G75.78+0.34 & 2019 & 952.0 & +37 & 17 & 02 & 4.1 & 5.29 \\
\hline
\end{tabular}

${ }^{1}$ Distances are from Churchwell et al. (1990) except G5.89 (Gomez et al. 1991; Zijlstra et al. 1990); G34.26, G31.41 and G45.47 (Kuchar \& Bania 1994); and G29.96 (Pratap et al. 1999).

2 Luminosities are from Churchwell et al. (1990), Hatchell et al. (2000) and Hofner et al. (1996), corrected for revised distances.

Section 2 of this paper describes the data set used to constrain the models. Section 3 contains the models of the dust continuum, and Sect. 4 those of the line emission. The results are discussed in Sect. 5. A follow-up paper (van der Tak \& Hatchell, in prep.) will present molecular abundances and their implications for the evolutionary status of these sources.

\section{Data selection}

The data which we use to constrain the physical structure falls into two categories: continuum and molecular line. For molecular line data, we use CS because (a) a wealth of CS measurements are available for these sources in the literature; (b) based on the models by Doty et al. (2002), we expect a simple chemistry with a constant abundance of CS throughout the cores. Molecules where the abundance varies with radius contain an additional free parameter; we need to constrain the density distribution first before we can investigate these cases.

There is also a great deal of continuum data available: ISO-LWS and IRAS far-infrared photometry; millimetre and submillimetre data at various wavelengths; and, critically, full submillimetre images from SCUBA which constrain the radial intensity profiles as well as the spectral energy distribution (SED) of these sources.

\subsection{Data on the SED}

The following data were used to constrain the spectral energy distribution.

- 850 and $450 \mu \mathrm{m}$ flux densities from SCUBA (JCMT) images (Hatchell et al. 2000; Thompson et al. 2002; Thompson et al., in prep.). We include flux densities within one beam $\left(9^{\prime \prime} / 14^{\prime \prime}\right)$ and integrated over the $2.5^{\prime}$ diameter map.

- SCUBA $1350 \mu \mathrm{m}$ single-pixel photometry (22" beam).

- ISO data were used in two ways: firstly, on the SED as an absolute flux density at $170 \mu \mathrm{m}$ with an assumed uncertainty of $30 \%$; and secondly, as the relative calibration of the ISO-LWS data is much better than $30 \%$, the shape of the ISO spectrum between 80 and $200 \mu \mathrm{m}$ was independently fitted.

- IRAS flux densities at 60 and $100 \mu \mathrm{m}$, taken from the HIRES processing of Hunter, who looked for FIR flux associated directly with the UCHII of interest (Hunter 1997). Fluxes are therefore less prone to confusion than those in the IRAS PSC (though fitting PSC data instead makes no difference to our conclusions). We assume uncertainties of $30 \%$. Fluxes are measured within $2^{\prime}$ of centroid.

- SHARC (CSO) $350 \mu \mathrm{m}$ flux densities: map sums and peak values, from Hunter et al. (2000) and Hunter (1997). 30\% uncertainties are assumed. Map sizes are $90^{\prime \prime}$ and the CSO beam at $350 \mu \mathrm{m}$ is $11^{\prime \prime}$.

We do not include in our fitting:

- Data at $\lambda>1350 \mu \mathrm{m}$, because these include a significant contribution from free-free emission, which we are not modelling.

- Data at $\lambda<60 \mu \mathrm{m}$. We know from previous work (Hatchell et al. 2000; van der Tak et al. 2000) that the $10 \mu \mathrm{m}$ silicate, $\operatorname{Br} \alpha, \operatorname{Br} \gamma$, and $\operatorname{Pf} \gamma$ optical depths and the mid-IR emission require lower column densities than the SED peak and the submm emission dictate. We believe these features and the related mid-IR continuum come from directions in which the source light escapes through relatively low optical depth paths. This breaks our assumption of spherical symmetry and introduces further parameters into the problem, which we are not attempting to incorporate into our model. By fitting the $\geq 60 \mu \mathrm{m}$ flux densities and submm SEDs, we are considering the majority of the mass in the system, which for our purposes of modelling molecular line emission is most important.

\subsection{ISO-LWS data}

The ISO archive $^{1}$ contains LWS01 spectra for all but three of our sources. These spectra cover the wavelength range 43$196 \mu \mathrm{m}$ at a resolution of $0.3-0.6 \mu \mathrm{m}$. The beam size of $80^{\prime \prime}$ is similar to the SCUBA and SHARC map sizes and the region used for the radial profiles (Sect. 3.2).

Because of the high brightness of these sources, the data need to be corrected for non-linearity of the detectors. To do this, data taken with 0.5 -second integrations had to be reprocessed at the ISO UK Data Centre, retaining only the first $0.25 \mathrm{~s}$ of each integration. For the strong source correction, Saturn was used as calibrator.

Further processing was done using the ISO Spectral Analysis Package (ISAP). Data were inspected and obviously outlying points removed. In particular, near the shortwavelength edge of detector SW5, a dip at $77 \mu \mathrm{m}$ was deleted. This feature is not seen in the data of detector SW4 and therefore considered spurious. The data were then averaged by detector, and corrected for instrumental fringing using standard algorithms.

\footnotetext{
1 http://www.iso.vilspa.esa.es
} 


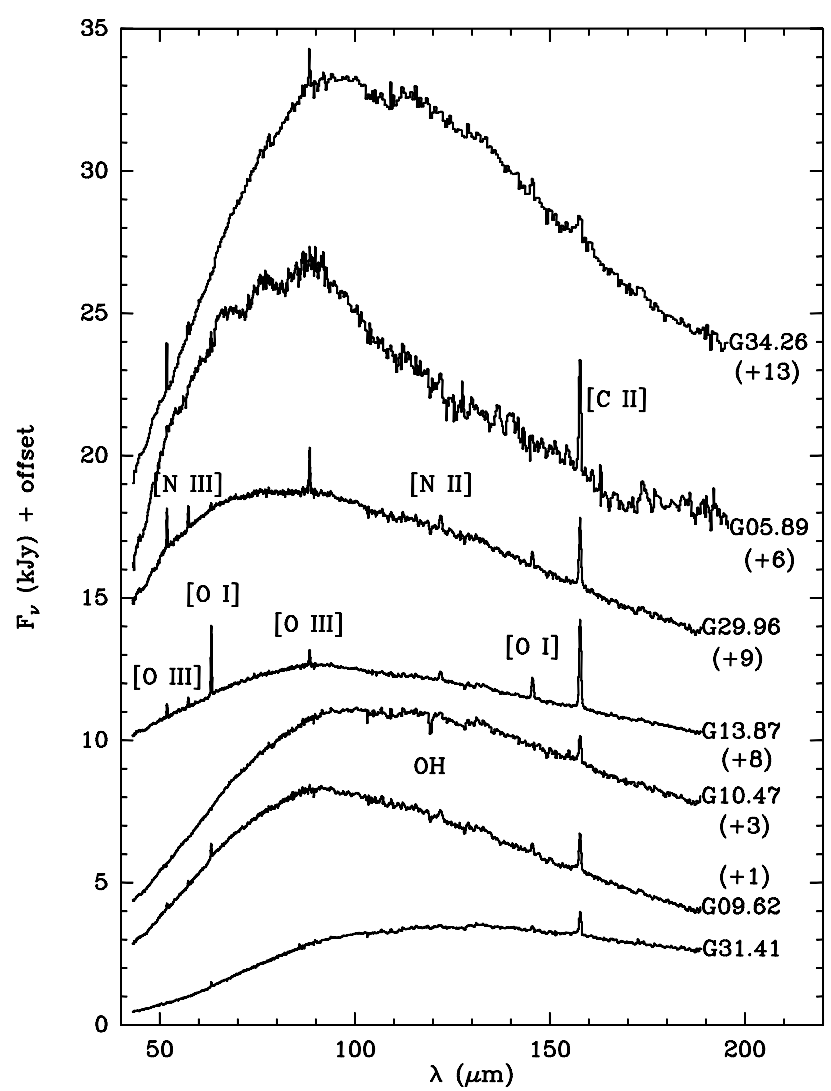

Fig. 1. ISO-LWS spectra of our sources. For clarity, the data are shifted vertically by the amount noted in brackets.

The data from the various detectors do not join smoothly. For strong sources, the differences are not caused by dark current subtraction problems, but by gain drifts, partly caused by the nonlinearity correction. In applying the gain shift, detector SW3 was used as reference.

Figure 1 shows the final spectra after averaging data from all detectors. Besides dust continuum, atomic and ionic finestructure lines are detected. These lines probably arise in the $\mathrm{H}$ II regions neighbouring the molecular regions studied here, and are not further discussed. The $\mathrm{OH}$ absorption against G10.47 may partly be formed in molecular gas and partly in a PDR, and will also not be discussed.

\subsection{Submm radial profiles}

We produced radial intensity profiles from the SCUBA images at 450 and $850 \mu \mathrm{m}$ as follows. Images at 850 and $450 \mu \mathrm{m}$ were produced from the SCUBA data by gridding from the irregular bolometer sampling onto a rectangular grid using linear interpolation. In order to avoid any reduction in resolution and later problems with radial averaging, a grid size of $1^{\prime \prime}$ was used, less than the bolometer sample spacing of $3.09^{\prime \prime}$ and far less than the JCMT resolution of $9^{\prime \prime} / 14^{\prime \prime}$. Variance data for the images were estimated by regridding at $3^{\prime \prime}$ spacing using a median algorithm which calculates the uncertainties $\sigma$ from the scatter on the data contributing to each pixel. Even for small numbers $N$ of datapoints in each bin, this gives a reasonable estimate of $\sigma: \sigma=\sigma_{0} \pm \frac{\sigma_{0}}{\sqrt{2(N-1)}}$ where $\sigma_{0}^{2}$ is the variance of the data in the bin.

For three sources this estimation of the uncertainties was not possible. Short integration times yielded one or fewer samples per pixel for G5.89 and G9.62. G75.78 was reduced as a scan map using tailored software.

Beam maps with uncertainties were produced in a similar way. As the $450 \mu \mathrm{m}$ beam is known to vary with elevation, two observations of Uranus at elevations appropriate to the source elevations were combined to produce the final beam map.

Radial intensity profiles of the sources were produced by taking a weighted average of the data in annuli centered on the image centroid spaced by $1 / 2$ beam. The uncertainties on the profiles were calculated from the variance data. For G5.89, G9.62 and G75.78, no variance data existed, and uncertainties on the profiles were estimated from the scatter of data within each annulus. In these cases the uncertainty is a measure of the deviation of the data from circular symmetry as well as measurement scatter. A better estimate of the annular mean, with smaller variance, can be made when variance data exists because one then weights towards more reliable data. The SCUBA noise is patchy, depending on bolometer feed. Neighbouring sources were masked out to sensible levels using circular masks in IRAF before radial averaging. The maps of G29.96 contain negative holes due to chopping onto nearby sources, which can be seen as a dip in the profiles (Fig. 6).

\subsection{CS data}

Our sources have been extensively observed in lines of CS and $\mathrm{C}^{34} \mathrm{~S}$, using the IRAM $30 \mathrm{~m}$ telescope for the $J=2 \rightarrow 1$, $3 \rightarrow 2$ and $5 \rightarrow 4$ lines, and the CSO and JCMT for the $7 \rightarrow 6$ and $10 \rightarrow 9$ lines. Figure 2 presents the data that we have used for our models. Most data are from Cesaroni et al. (1991), Churchwell et al. (1992) and Olmi \& Cesaroni (1999), with additions from Plume et al. (1992, 1997). Beam sizes vary between $11^{\prime \prime}$ and $25^{\prime \prime}$ and are taken into account in the modelling.

\section{Continuum modelling}

In order to determine radial density and temperature distributions, total column density, and other properties of the sources we compare the observed SED and submm radial intensity profiles with the results of the radiative transfer code DUSTY (Ivezić \& Elitzur 1997; Ivezić et al. 1997). This publicly available program ${ }^{2}$ exploits scaling properties to reduce the number of independent parameters in the spherical radiative transfer problem. We discuss the input parameters below in Sect. 3.1. Some further processing is required before the output of DUSTY can be compared with the observations, as discussed in Sect. 3.2.

Spherical symmetry is a first approximation to these sources. However, apart from G9.62 and G75.78, axis ratios based on the $850 \mu \mathrm{m}$ maps show no significant deviation from circular symmetry which would necessitate a more complex model. Thus we keep the number of parameters to a minimum,

\footnotetext{
${ }^{2}$ http://www.pa.uky.edu/ moshe/dusty
} 

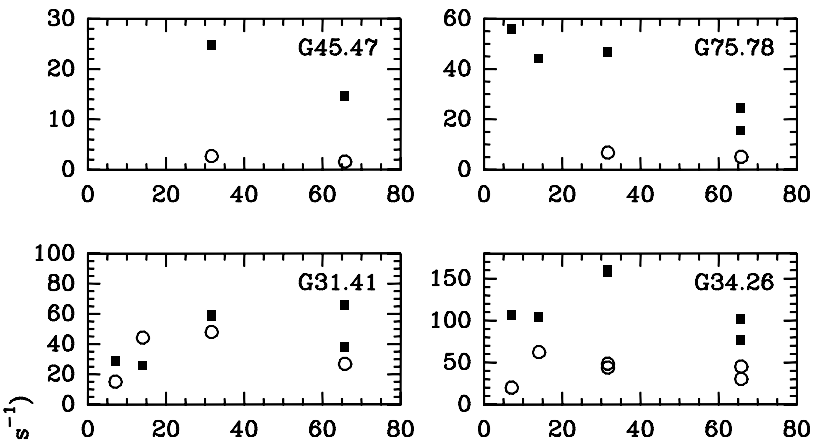

g
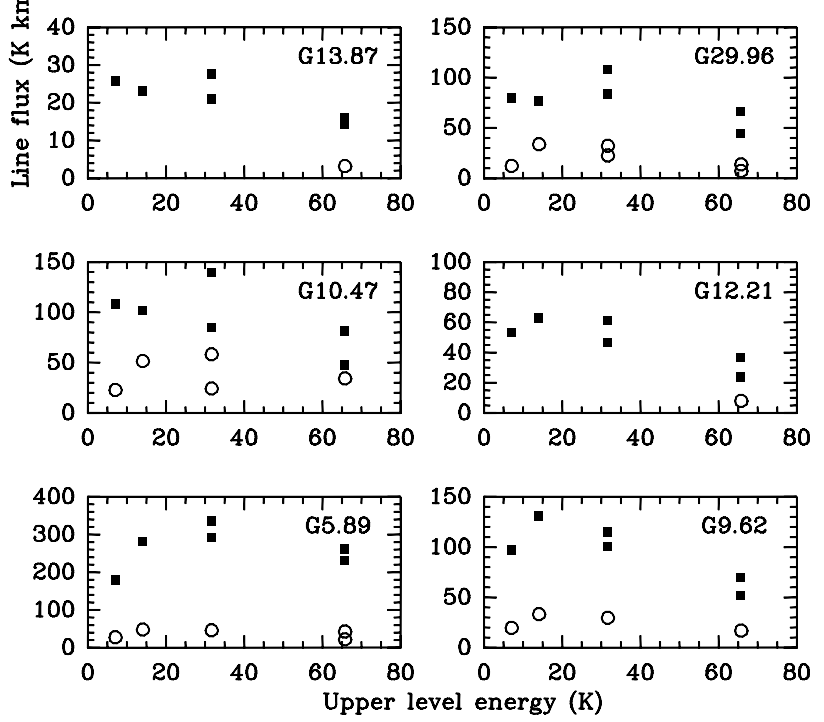

Fig. 2. Emission line data for $\mathrm{CS}$ (filled squares) and $\mathrm{C}^{34} \mathrm{~S}$ (open circles).

both to restrict the parameter space to model and so that we can hope to constrain the parameters with the available data. A spherical description of the density and temperature distribution is sufficient for further modelling of molecular line emission.

\subsection{Model parameters}

The inputs to DUSTY are given in our case as: the optical depth at $100 \mu \mathrm{m}, \tau_{100}$; density power law index $p$; the inner radius $r_{\mathrm{im}}$; the stellar black body temperature $T_{\star}$; and the shell thickness (ratio of outer and inner radii) $Y$. In addition, the dust optical properties are specified. This small number of parameters completely defines the radiative transfer problem. The output then scales by the luminosity to fit individual sources.

- Dust type. As dust model we use the coagulated, icy grains of Ossenkopf \& Henning (1994; Model 5), shown to be appropriate for star forming regions by van der Tak et al. (1999). Ossenkopf \& Henning list the absorption coefficients for their ices between 1-1300 $\mu \mathrm{m}$. Visual and ultraviolet opacities are from Pollack et al. (1994; Model 1). Scattering coefficients are calculated from the absorption coefficients using albedos from Draine \& Lee (1984), but tests show that for our problems the scattering coefficients are unimportant.
- Inner radius. $r_{\text {in }}$ The inner radius was fixed at $3.28 \times$ $10^{15} \mathrm{~cm} \times \sqrt{L / 10^{4.0}}\left(0.1-0.4^{\prime \prime}\right)$. Dust temperatures at the inner radius vary from 490 to $600 \mathrm{~K}$. Previous work suggested that the highest temperatures that dust reaches in these sources are a few hundred $\mathrm{K}$, much lower than the dust sublimation temperature of $\approx 1500 \mathrm{~K}$. Molecular gas is observed at these temperatures in several of these sources (Hatchell et al. 1998; Cesaroni et al. 1998; Wyrowski et al. 1999).

- Driving source temperature $T_{\star}$. We take a black body input at $42000 \mathrm{~K}$, equivalent to an $\mathrm{O} 6$ star. The exact temperature of the input spectrum is not important as the infrared and $\mathrm{mm} / \mathrm{submm}$ emission is all reprocessed light.

- Optical depth $\tau$. From the position of the SED peak, we know that $\tau=1$ at a few $100 \mu \mathrm{m}$. We consider models with $\tau_{100}=0.1,0.5,1.0,2.0,3.0$, and 4.0. Higher optical depth models would be useful (see Sect. 5.2), but are beyond our current computing limits (models are being run on a Linux cluster of four dual-CPU $1 \mathrm{GHz}$ machines with 2 GB of memory each). A second determinant of the optical depth is that in the optically thin regime, the brightness should be of the order $\tau \times B_{v}$, where $B_{v}$ is the Planck function. Submillimeter observations suggest that $\tau_{100} \gtrsim 1$.

- Density power law index $p$. In contrast to the low mass case, there is no clear prediction from theory for the density laws expected in the envelopes of massive SFRs. For this reason we consider only the simplest power law density laws. Values of $p$ from 1.25 to 2.25 have been tried, based on previous experience (Hatchell et al. 2000; van der Tak et al. 2000; Mueller et al. 2002).

- Shell thickness $Y$. The ratio of outer to inner radii. We consider a wide range of values from smaller than the map sizes to larger: $Y=100,200,300,600$, and 1200 .

- External radiation. We assumed the Black-Draine interstellar radiation field of Evans et al. 2001 (see references therein).

\subsection{DUSTY outputs and post-processing}

DUSTY computes radial intensity profiles at selected wavelengths assuming a luminosity of $10^{4} L_{\odot}$. Although the solution of the radiative transfer problem does not depend on the luminosity, in the post-processing we have to scale the output to the luminosity and distance of each source in order to take finite beam and map sizes into account.

For many of our sources, only kinematic distances are available, which are uncertain by factors of up to two. In the future, near-infrared spectroscopy (e.g., Watson \& Hanson 1997; Kaper et al. 2002) may provide more accurate distances. However, because of the luminosity scaling, our model results are independent of distance. Note that we assume that all of the stellar radiation is absorbed by the dust, and that a negligible fraction goes into ionizing an HII region.

The results are only weakly dependent on the luminosity, which we hold fixed. Changing the luminosity primarily scales the fluxes, which affects the SED. Secondly, it changes the radial scale $(r \propto \sqrt{L})$, which affects both the radial profiles and 

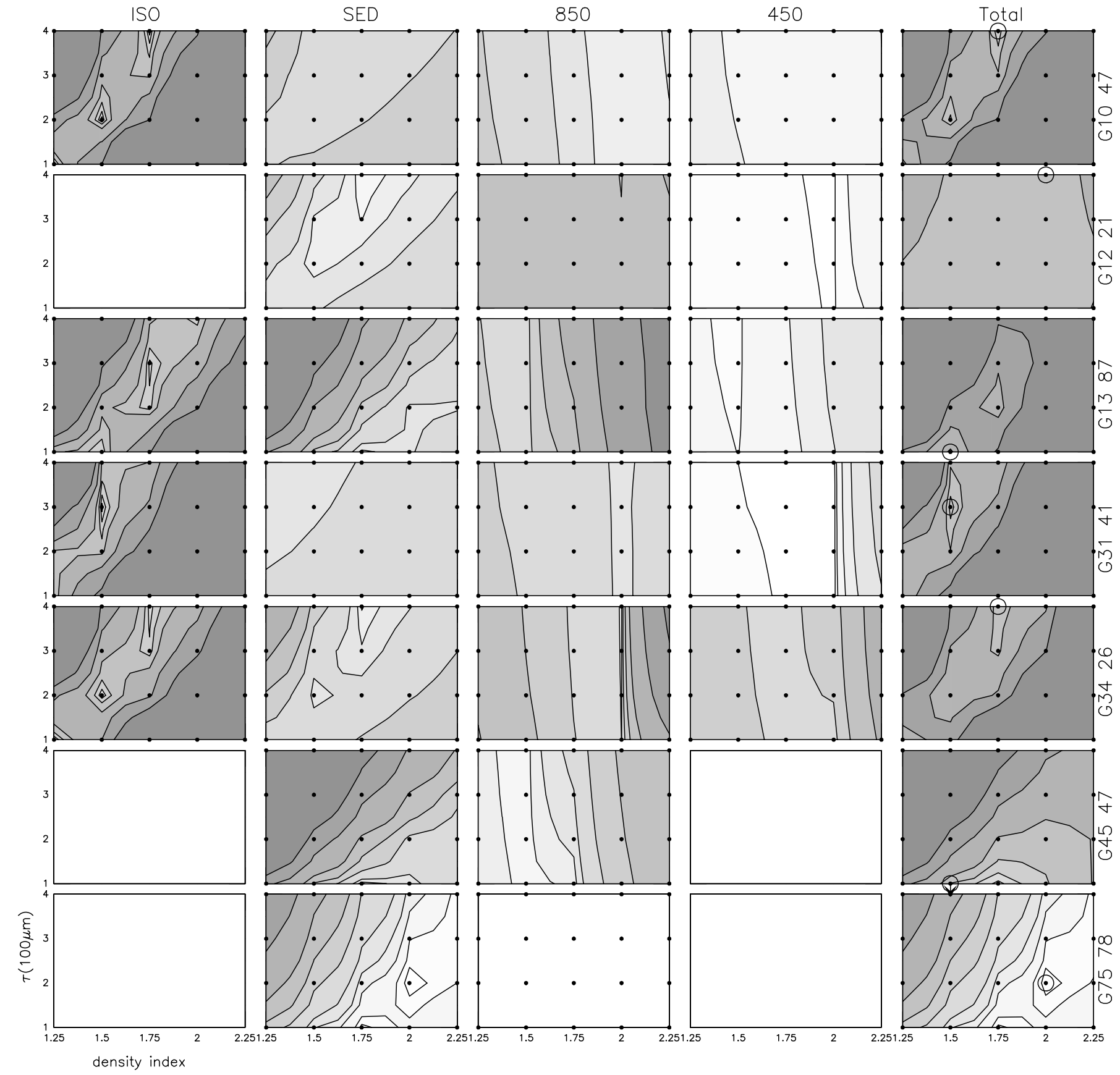

Fig. 3. Continuum model fits: non-reduced $\chi^{2}$ contours vs. $p$ and $\tau$ for the $7 / 10$ sources where the best fit favours a large shell size $(Y=600$ is displayed). Left to right, $\chi^{2}$ is displayed for fits to: the ISO-LWS spectral shape between 60 and $180 \mu \mathrm{m}$; the SED (absolute flux densities from various instruments); 850 and $450 \mu \mathrm{m}$ radial intensity profiles from SCUBA; and the combined $\chi^{2}$ for all datasets. Contours/greyscale steps are, white to black, 10, 20, 50, 100, 200, 500,1000, 2000, 5000, 10000 . Model points are marked as dots and the best fits are marked as circles on the total $\chi^{2}$ plots. Sources where the models favoured a small shell size are in Fig. 4.

the ISO fits. But this second effect is small. As shown below, it is the ISO spectrum which dominates the determination of the best fit, and even luminosity changes of a factor of 2 make little change to the overall results.

For model fluxes to compare with the SED and ISO data, we extract from the DUSTY radial intensity profiles flux densities within Gaussian beams or integrated out to some outer radius, to match the observations.
To derive model profiles, the radial intensity distributions at 450 and $850 \mu \mathrm{m}$ were turned into $2 \mathrm{D}$ synthetic images and convolved with the beam (as measured on Uranus: Sect. 2.3). Telescope chopping (beam or wobbler switching to subtract sky emission) was simulated by shifting the image by the $2^{\prime}$ chop throw in each direction and subtracting. Profiles were then extracted by radial averaging in the same way as for the data (Sect. 2.3). Because of the uncertainty in the beam 


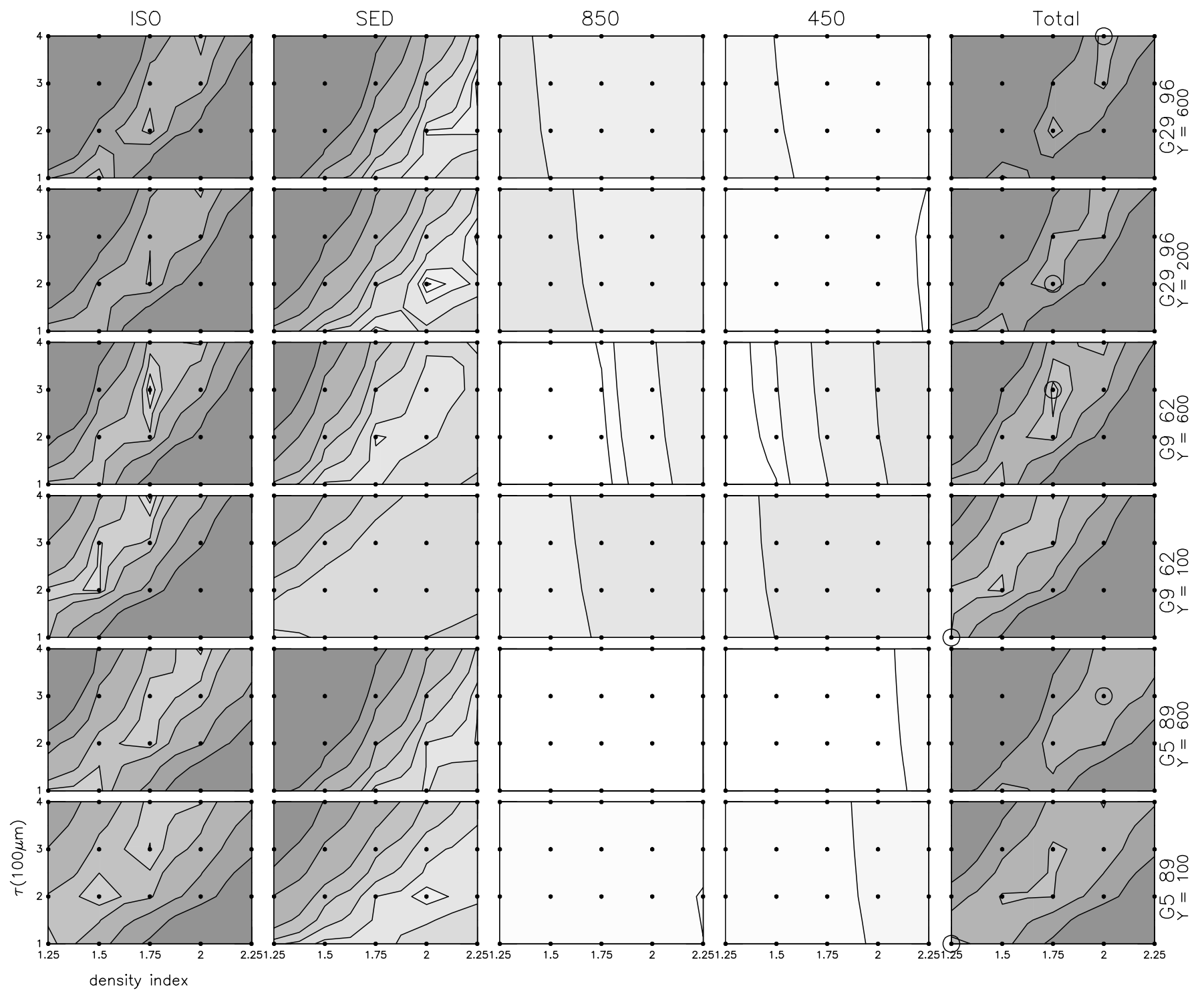

Fig. 4. Continuum model fits: $\chi^{2}$ contours vs. $p$ and $\tau$ for the $3 / 10$ sources where the best fit favours a small shell size, but is poorly constrained by the profiles. Both the best fit shell size and $Y=600$ are displayed. Other details as Fig. 3.

measurement, the model profiles also have associated uncertainties, which are small compared to those on the source profiles, but nonetheless are taken into account by adding the uncertainties in quadrature. Fits to profiles were made between $20^{\prime \prime}$ and $50^{\prime \prime}(0.36-1.2 \mathrm{pc}$ at $5 \mathrm{kpc})$, beyond one beam and avoiding self-chopping effects at the map edges.

\subsection{Results}

We performed a least-squares analysis to determine which models best fit the data. We calculated $\chi^{2}$ values for the 60 $1300 \mu \mathrm{m}$ flux densities, the shape of the ISO spectrum, the 450 and $850 \mu \mathrm{m}$ radial profiles, and for the combined dataset. The total number of datapoints, best fit parameters and reduced $\chi^{2}$ for each source are given in Table 2 , along with the mass within $1 \mathrm{pc}$.

Figures 3 and 4 display the $\chi^{2}$ plots for our models compared to the four datasets (ISO data, total SED, 850 and $450 \mu \mathrm{m}$ profiles) and the combined likelihood of fitting all the data. The best fits are marked with a circle, though these should be considered cautiously as the region of parameter space allowed by the data can be quite large. We have not given formal parameter errors calculated from $\Delta \chi^{2}$ contours. These would misrepresent the range of acceptable parameters, partly because of the coarse model grid, and partly because there are more uncertainties than the formal fit errors take into account, borne out by reduced $\chi^{2}>1$. Figure 5 compares the overall best fit models with the ISO spectra, Fig. 6 with the radial profiles, and Fig. 7 with the SED.

\section{CS modelling}

To test the density structures derived from the continuum data, models were made of emission lines. The CS molecule, with its large mass and large dipole moment, is an especially sensitive tracer of density, with lines at observable frequencies that span a wide range of critical densities. In addition, depletion of CS 


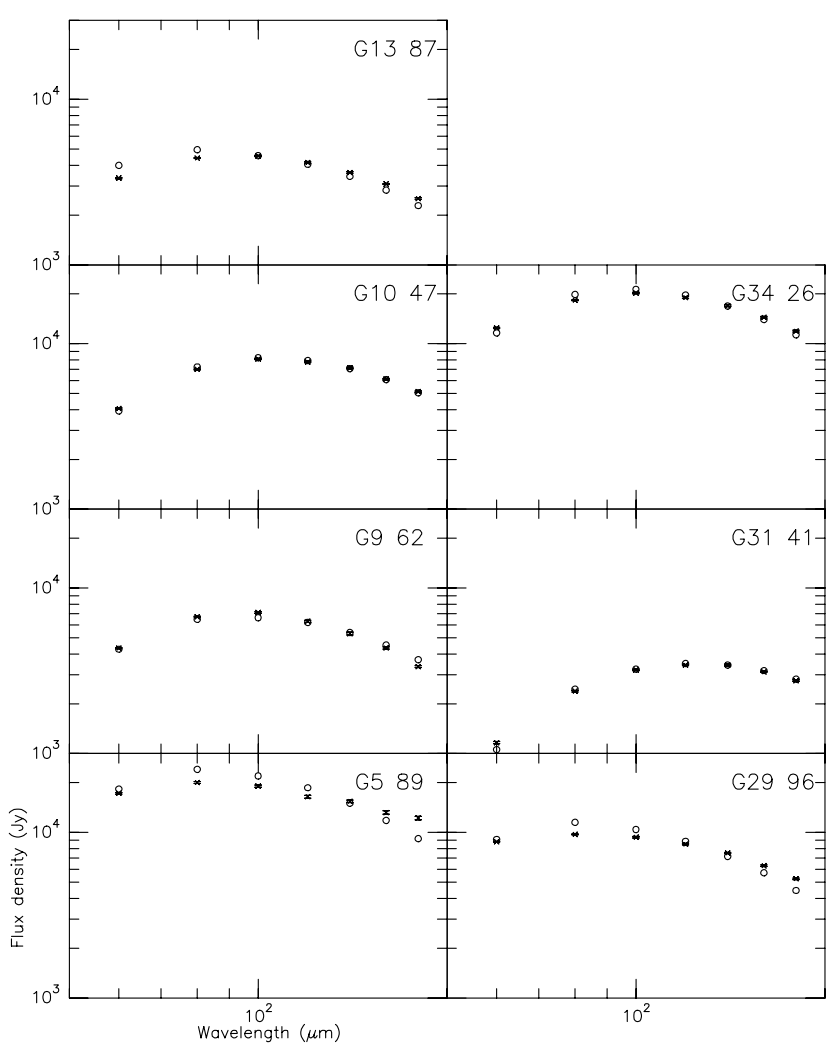

Fig. 5. ISO-LWS spectra (crosses) overlaid with the best continuum model fits (circles).

Table 2. Best fit continuum models: size, density index (from $850 \mu \mathrm{m}$ profile only and from overall fit), optical depth at $100 \mu \mathrm{m}$, reduced $\chi^{2}$, and mass within 1 pc. For G5.89, G9.62 and G29.96, where the best fits imply a low $Y$ outer cutoff, we have also given the results for $Y=$ 600; see discussion.

\begin{tabular}{ccccccc}
\hline \hline Source & $Y$ & $p$ & $\tau_{100}$ & $\chi^{2}$ & $\begin{array}{c}M_{1 \mathrm{pc}} \\
M_{\odot}\end{array}$ & $p_{\text {prof }}$ \\
\hline G5.89 & 100 & 1.25 & 1 & 37 & 1988 & \\
& 600 & 2.00 & 3 & 70 & 195 & 2.25 \\
G9.62 & 200 & 1.25 & 1 & 7 & 2414 & \\
& 600 & 1.75 & 3 & 22 & 1897 & 1.50 \\
G10.47 & 1200 & 1.75 & 4 & 12 & 2518 & 2.00 \\
G12.21 & 600 & 2.00 & 4 & 45 & 1537 & 2.00 \\
G13.87 & 300 & 1.50 & 1 & 43 & 905 & 1.50 \\
G29.96 & 200 & 1.75 & 2 & 79 & 1631 & \\
& 600 & 2.00 & 4 & 104 & 726 & 2.00 \\
G31.41 & 600 & 1.50 & 3 & 20 & 2657 & 2.00 \\
G34.26 & 200 & 1.75 & 4 & 32 & 2365 & 2.00 \\
G45.47 & 600 & 1.5 & 0.5 & 6 & 821 & 1.50 \\
G75.78 & 1200 & 2.00 & 2 & 0.4 & 361 & 2.00 \\
\hline
\end{tabular}

on grains should be minimal at the temperatures present in our sources (Sect. 5.1).

We have used the publicly available ${ }^{3}$ Monte Carlo radiative transfer program by Hogerheijde \& van der Tak (2000) to model CS integrated intensity at source centre. Radial

\footnotetext{
${ }^{3}$ http://talisker.arizona.edu/ michiel/ratran.html
}

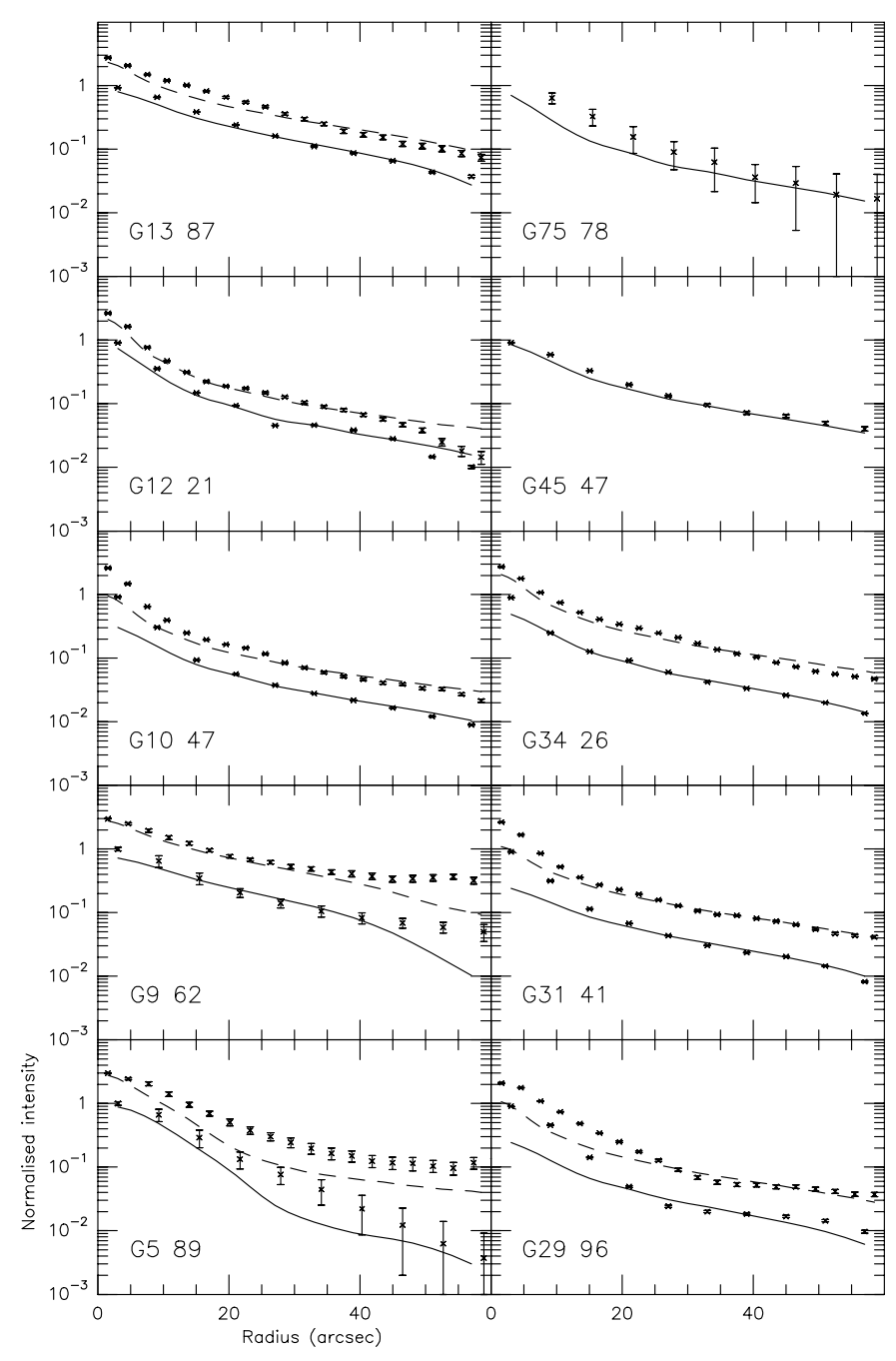

Fig. 6. Normalised radial intensity profiles at 850 and $450 \mu$ m overlaid with the best continuum model fits. The $450 \mu \mathrm{m}$ profiles are multiplied by 3 .

Table 3. Results of CS models.

\begin{tabular}{lrccc}
\hline \hline Source & $p$ & $\chi^{2}$ & $N^{a}$ & $\mathrm{CS} / \mathrm{H}_{2}$ \\
\hline G5.89 & 2.25 & 2.93 & $6+5$ & $1 \times 10^{-6}$ \\
G9.62 & 1.50 & 1.80 & $6+4$ & $2 \times 10^{-8}$ \\
G10.47 & 2.00 & 0.80 & $6+5$ & $1 \times 10^{-7}$ \\
G12.21 & 1.25 & 0.92 & $6+1$ & $2 \times 10^{-8}$ \\
G13.87 & 1.25 & 1.60 & $6+1$ & $2 \times 10^{-9}$ \\
G29.96 & 1.50 & 1.49 & $6+6$ & $2 \times 10^{-8}$ \\
G31.41 & 2.00 & 1.41 & $6+4$ & $5 \times 10^{-7}$ \\
G34.26 & 2.25 & 1.78 & $6+6$ & $1 \times 10^{-7}$ \\
G45.47 & 1.25 & 1.09 & $2+2$ & $2 \times 10^{-9}$ \\
G75.78 & 1.75 & 1.49 & $6+2$ & $1 \times 10^{-8}$ \\
\hline
\end{tabular}

${ }^{a}$ Number of $\mathrm{CS}$ and $\mathrm{C}^{34} \mathrm{~S}$ lines used in the fit.

profiles of density and temperature were taken from DUSTY's output. The kinetic and dust temperatures were assumed to be equal since the densities of our sources exceed $10^{4} \mathrm{~cm}^{-3}$. The number of radial shells ranged from 29 to 78 with increasing dust optical depth (determined by DUSTY). Turbulent line 


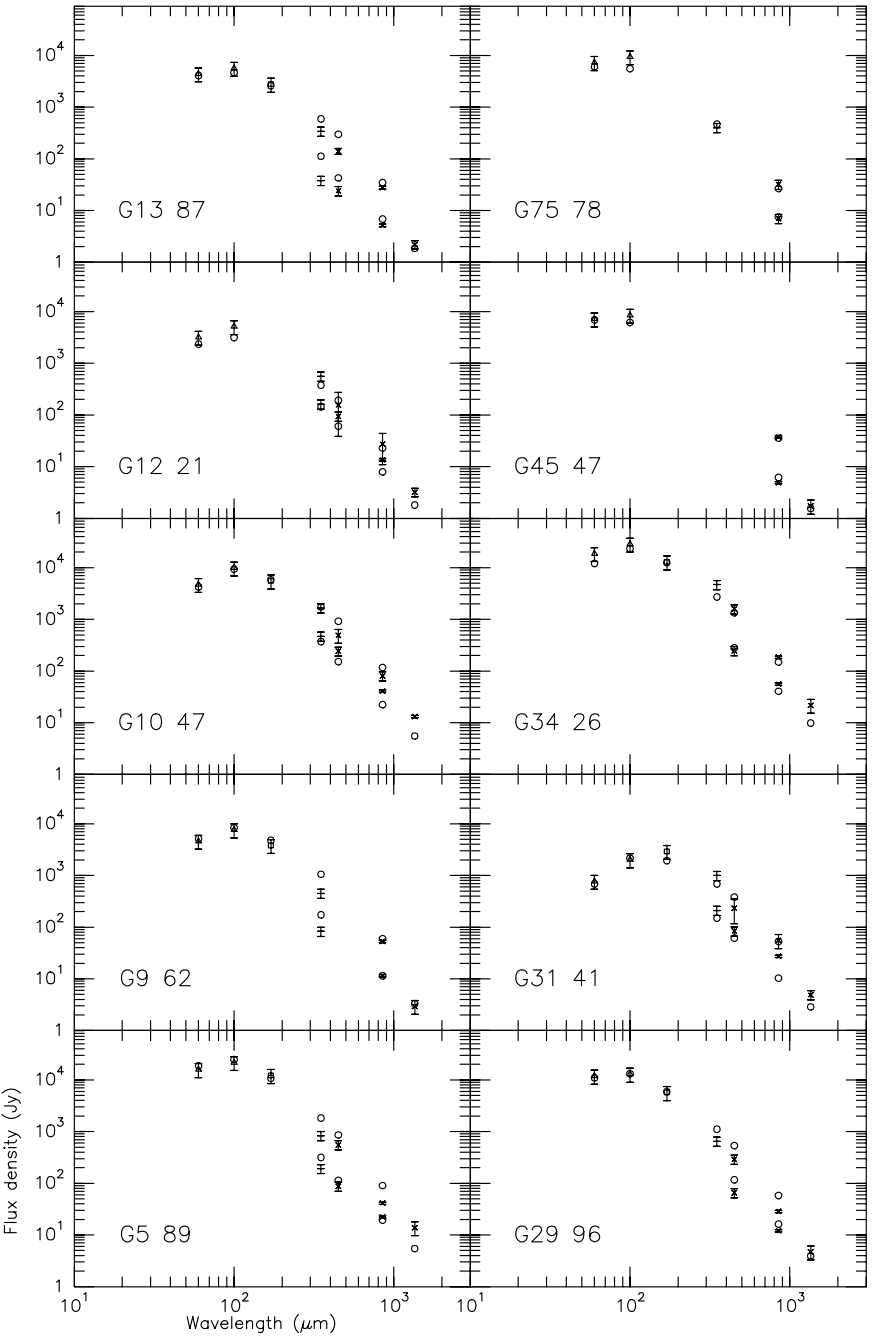

Fig. 7. Flux densities from IRAS HIRES (triangles) ISO (squares); SHARC $(+)$; and SCUBA $(\times)$, overlaid with the best continuum model fits (circles).

widths are taken from $\mathrm{C}^{34} \mathrm{~S}$ data (Sect. 2.4); thermal broadening is included but negligible. The radiative transfer model includes the 21 lowest rotational states of CS, with term energies, statistical weights and Einstein $A$ coefficients taken from the JPL catalogue ${ }^{4}$ (Pickett et al. 1998). Collisional rate coefficients are taken from Turner et al. (1992) ${ }^{5}$. Radiative excitation of rotational lines by dust and by the microwave background is also taken into account, again using grain properties from Ossenkopf \& Henning (1994).

Most models were run for G10.47, and Fig. 8 shows the results. For each value of the dust optical depth at $100 \mu \mathrm{m}$ $\tau_{100}$ and density law index $p$, the model with the best-fitting $\mathrm{CS}$ abundance is plotted. As the figure shows, the CS lines are sensitive to the density index, almost irrespective of $\tau_{100}$ which measures total column density. Therefore, for the other sources, models were only run for $\tau_{100}=3$, and Table 3 lists the results.

\footnotetext{
${ }^{4}$ http://spec.jpl.nasa.gov

${ }^{5}$ see http://www.giss.nasa.gov/data/mcrates
}

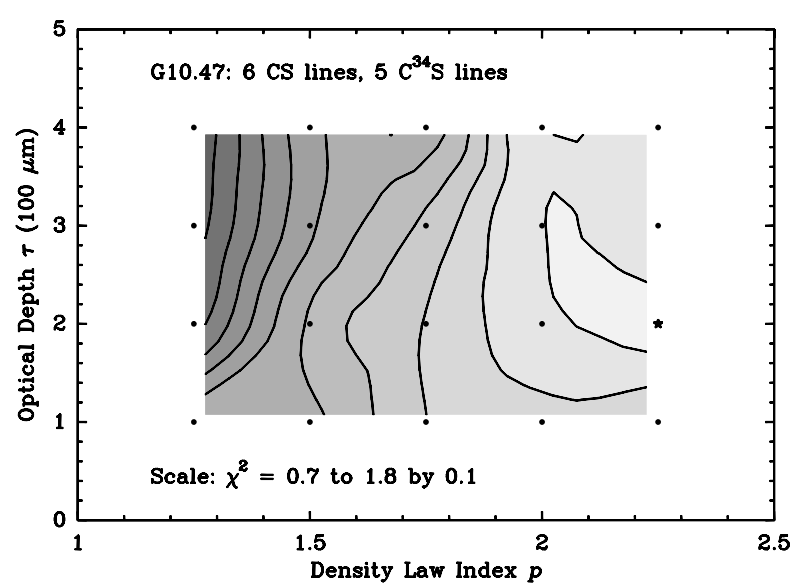

Fig. 8. Quality parameter $\chi^{2}$ of the fits to the CS lines in G10.47, as a function of $\tau_{100}$ and $p$.

\section{Discussion}

\subsection{Outer cutoffs?}

For most sources, small shell models $(Y \leq 200)$ are ruled out by the intensity distributions, which do not show an outer cutoff. Only three sources with poorly constrained profiles (due to non-sphericity (G29.96) or poor signal-to-noise (G5.87, G9.62)) favour small shell models. For these we have shown both the $Y=600$ and best $-Y \chi^{2}$ plots (Fig. 4). We believe that if the profiles for these sources could be better constrained (for G5.87 and G9.62 by longer submm integrations) then the small shell models would be ruled out for these sources also.

For large outer radii, the intensity profile approaches a power law with index $m$, which in the infinite-shell limit is given by $m \simeq p-0.5$ at $850 \mu \mathrm{m}$ (Adams 1991). In this case, the map size acts as lower limit to the outer radius of the model. Otherwise, as the effect of changing $Y$ on the profile steepness above $Y=200$ is equivalent to changing $p$ by $<0.1$, the profiles do not differentiate strongly between different $Y$, and neither do the spectral energy distributions as the effect of adding more cold material at large radius is minimal.

Temperatures at the outer edge varied from 15 to $27 \mathrm{~K}$, with higher temperatures for models with a small radius cutoff. This is another reason to prefer large-radius models, as lower temperatures better match the surrounding molecular cloud material.

\subsection{Constraints on $\tau$ and $p$}

From the SED and ISO fits, there is a degeneracy between optical depth $\tau$ and density index $p$. Both high optical depth and a flatter density distribution act to produce more colder material which emits at longer wavelength.

This degeneracy is partially broken by the radial profile fits, which constrain $p$ but are largely independent of $\tau$ (Figs. 3, 4, Table 2). The profiles indicate a preference for low $p$ (G9.62, G13.87, G45.47) or high $p(\mathrm{G} 10.47, \mathrm{G} 12.21, \mathrm{G} 29.96, \mathrm{G} 31.41$, G34.26, G75.78). If low shell size models are ruled out in the continuum fits (see Sect. 5.1 above), then G5.89 joins the list of steep profile sources. However, in the total $\chi^{2}$ plots, the profiles 
do not constrain $p$ tightly enough to outweigh the influence of the SED fits: there are not enough profile points and they are too uncertain. This is despite good signal-to-noise measurement of the profiles in most cases: errorbars result from the real-life asymmetry of the sources as well as measurement noise.

Considering just the profile fits, the strong molecular hot core sources (G5.89, G10.47, G12.21, G29.96, G31.41, G34.26 and G75.78) have steep density distributions ( $p \geq 1.75)$ whereas the weak molecular line emitters G13.87 and G45.47 have flatter profiles $(p=1.50)$, as Hatchell et al. (2000) discovered. If one looks at the joint $\chi^{2}$, dominated by the SED and ISO fits, the case is less clearcut with some hot cores (in particular G31.41) showing low $p$. It may be that a higher optical depth model ( $\tau>4$, which we were unable to compute) could yield a higher $p$ solution which would reconcile the profile and SED results in these cases.

The $\tau-p$ degeneracy is further broken by the CS results. The CS fits are largely independent of $\tau$ but depend strongly on $p$. The CS fits are in general agreement with the profile fits (within \pm 0.25 ): again, G13.87 and G45.47 have flatter profiles $p=1.25$ whereas hot core sources (G5.89, G10.47, G31.41, G34.26, G75.78) have steeper profiles ( $p \geq 1.75)$. G12.21 and G29.96 are the only sources for which the CS results ( $p=1.25$ and $p=1.50)$ and profile results $(p=2.0)$ cannot easily be reconciled, perhaps due to the great distance of G12.21 (13.5 kpc) and the negative holes in the G29.96 map.

Comparing the CS results with the overall continuum fits shows agreement within $p \pm 0.5$ : again, higher $p$, higher $\tau$ solutions for G34.26 and G31.41 would allow a better match between continuum and CS results.

From all the fits, the parameters are constrained to $p \pm 0.25$, $\tau \pm 1$ (given $p)$; and $Y \geq 300$.

\subsection{Low-p sources missing hot cores}

It is clear from the $\chi^{2}$ contours that G45.47 and G13.87 (both $p=1.25-1.50)$ are low optical depth sources that cannot be fitted with higher $\tau$, higher $p$ solutions. This has not compromised their ability to form stars.

These sources also show little evidence for any hot molecular core, with no $\mathrm{CH}_{3} \mathrm{OH}$ above $100 \mathrm{~K}$, and no chemical daughter products such as $\mathrm{CH}_{3} \mathrm{CN}$ detected, although in both cases $\mathrm{NH}_{3}(4,4)$ transitions $200 \mathrm{~K}$ above ground were detected (Hatchell et al. 1998; Cesaroni et al. 1992).

An explanation for both $p \sim 1.5$ density profiles and lack of hot molecular cores in these sources could be that they are infalling with collapse timescales less than the $10^{4}$ years necessary for chemical processing (Rodgers \& Charnley 2003). G45.47+0.05 shows no evidence for infall, with apparent $\mathrm{P}$ Cygni profiles in $\mathrm{NH}_{3}$ due not to infall but to multiple molecular components (Wilner et al. 1996).

Alternatively, the lack of apparent hot core activity can be simply explained by a combination of the lower column density and flatter density distribution. The masses at small radius where grain evaporation occurs (roughly $T>100 \mathrm{~K}$ ) are smaller by a factor of $\sim 5$ in a $\tau=1, p=1.5$ source compared to $\tau=4, p=2$ typical of the strong hot core sources. This is enough to put most of the molecular lines used to trace hot cores below the detection limit of the earlier studies, but with better sensitivity they should be seen. For example, with rms noise of $20 \mathrm{mK} T_{\mathrm{A}}^{*}$ rather than $50 \mathrm{mK}$ (Hatchell et al. 1998), $\mathrm{CH}_{3} \mathrm{CN}$ at $239 \mathrm{GHz}$ and $\mathrm{CH}_{3} \mathrm{OH}$ at $241.8 \mathrm{GHz}$ should be detected. If daughter species such as $\mathrm{CH}_{3} \mathrm{CN}$ were detected, infall would definitely be ruled out.

A likely explanation for the relative lack of molecular gas and dust at small radius is that star formation has used up or disrupted more of the available material. Either G13.87 and G45.47 are more evolved than their steep-index counterparts, or they started with less gas in the first place.

\subsection{Central cores?}

Does the density law steepen at small radius? This would show up as excess flux at small radius in the profiles. Although the overall best fits (Fig. 6) leave a flux excess in the centre for the hot core sources G10.47, G31.41 and G29.96, the steeper density indices of both the profile and CS best fits leave no excess central flux, i.e., a single power law model adequately fits the data without a central core. Interferometer (BIMA) data at $1.2 \mathrm{~mm}$ and $3^{\prime \prime}$ resolution for the four hot core sources $\mathrm{G} 10.47$, G29.96, G31.41, and G34.26 shows that the power laws continue smoothly into the inner regions of the hot cores down to $r \sim 2 \times 10^{16} \mathrm{~cm}$, with no dust emission seen at smaller radius (F. Wyrowski, in prep.). This rules out fitting the continuum data with a lower- $p$ envelope plus compact submm-emitting core (Hatchell et al. 2000). The lack of submm emission in the core centres could be due to either optically thick cores or a central cavity (Wyrowski, in prep.; van der Tak et al. 2000). Note that the UCHII which are assumed to contain the sole heating sources in our modelling and must be surrounded by a cavity, are separated from the dust emission peaks at the resolution of BIMA.

\subsection{Effect of optical properties}

There are some differences between the best fits to the ISO data (where available) and the submillimeter data. In 4/7 cases, the ISO data indicate a higher $\tau$ or lower $p$, i.e., an SED which peaks at longer wavelength. In these cases, the ISO and submillimeter points might be reconciled by reducing the long wavelength emissivity of the dust by a factor of $2-3$. Of the Ossenkopf \& Henning 1994 models, only the thick ice models reduce $\kappa_{500} / \kappa_{100}$ (models $7-9$ ). Thick ice is unlikely in the warm inner regions where ices evaporate but the bulk of the submm emission comes from cold dense outer regions which may have thick ices. The limited dataset is not sufficient to make this a firm conclusion. With submillimetre data at more wavelengths one could estimate the dust emissivity and therefore the ice thickness for individual sources, assuming this were constant throughout the core. In practice, the ice thickness and composition is likely to vary with radius. In only one source do the far-infrared data indicate a lower $\tau$ (or higher $p$ ) than the millimeter points. 


\section{Conclusions}

We have constructed models of the envelopes of ten embedded massive stars. The main assumptions are spherical symmetry and central heating. Our models are constrained by lowresolution $(R \sim 500)$ far-infrared and submillimeter spectra in 9-80" beams, maps of submillimeter emission at 9-15" resolution, and emission lines of CS and $\mathrm{C}^{34} \mathrm{~S}$ measured in $10-30^{\prime \prime}$ beams. Our conclusions are the following.

- On scales of 10-60", or $0.25-1.55 \mathrm{pc}$ for the mean distance of $5.3 \mathrm{kpc}$, the envelopes of embedded massive stars are well described by models with a power law density structure. Power law indices are uniformly distributed between 1.25 and 2.25, similar to the range found for other samples (van der Tak et al. 2000).

- The sources with the steepest density gradients are richest in molecular line emission as found before (Hatchell et al. 2000). A model with shallow gradients plus central "cores" is an alternative for the hot core sources, but cores are not required by these data: a pure power law is sufficient. Evidence for distributed heating sources is seen nowhere.

- G13.87 and G45.47 (non-hot-core sources) have low optical depth and shallow density profiles, probably because star formation has used up more of the material in the inner regions. Their lack of detected hot core molecular line emission can be explained by the lower masses at small radius.

- The density power law indices for radial profiles and emission lines agree well (by $p \pm 0.25$ ), while the continuum spectrum sometimes deviates (by $p \pm 0.5$ ). These error bars and deviations are not set by data quality, but are the limitations of our modeling approach, in particular the assumption of spherical symmetry. Robust models require use of all these kinds of data, and the detailed treatment that we have outlined.

Acknowledgements. This work is partly based on observations with ISO, an ESA project funded by ESA member states (especially the PI countries: France, Germany, The Netherlands and the UK), with participation of ISAS (Japan) and NASA (United States). We thank Sabine Philipp and Sarah Leeks for help with the ISO data reduction, Mark Thompson for reduced SCUBA maps, Malcolm Walmsley and Moshe Elitzur for useful discussions, Maia Nenkova for DUSTY support, and Neal Evans for the ISRF.

\section{References}

Adams, F. C. 1991, ApJ, 382, 544

André, P., Ward-Thompson, D., \& Barsony, M. 2000, in Protostars and Planets IV, ed. V. Mannings, A. P. Boss, \& S. S. Russell (Tucson: University of Arizona Press), 59

Beuther, H., Schilke, P., Menten, K. M., et al. 2002, ApJ, 566, 945

Cesaroni, R., Walmsley, C. M., Koempe, C., \& Churchwell, E. 1991, A\&A, 252, 278

Cesaroni, R., Hofner, P., Walmsley, C. M., \& Churchwell, E. 1998, A\&A, 331, 709

Cesaroni, R., Walmsley, C. M., \& Churchwell, E. 1992, A\&A, 256, 618

Churchwell, E., Walmsley, C. M., \& Cesaroni, R. 1990, A\&AS, 83, 119
Churchwell, E., Walmsley, C. M., \& Wood, D. O. S. 1992, A\&A, 253, 541

Doty, S. D., van Dishoeck, E. F., van der Tak, F. F. S., \& Boonman, A. M. S. 2002, A\&A, 389, 446

Draine, B. T., \& Lee, H. M. 1984, ApJ, 285, 89

Evans, N. J., Rawlings, J. M. C., Shirley, Y. L., \& Mundy, L. G. 2001, ApJ, 557, 193

Gibb, A. G., Wyrowski, F., \& Mundy, L. G. 2003, in Chemistry as a diagnostic of star formation (NRC), in press

Gomez, Y., Rodriguez, L. F., Garay, G., \& Moran, J. M. 1991, ApJ, 377, 519

Hatchell, J., Thompson, M. A., Millar, T. J., \& MacDonald, G. H. 1998, A\&AS, 133, 29

Hatchell, J., Fuller, G. A., Millar, T. J., Thompson, M. A., \& Macdonald, G. H. 2000, A\&A, 357, 637

Hofner, P., \& Churchwell, E. 1996, A\&AS, 120, 283

Hogerheijde, M. R., \& van der Tak, F. F. S. 2000, A\&A, 362, 697

Hunter, T. R. 1997, A Submillimeter Imaging Survey of Ultracompact HII Regions, Ph.D. Thesis (California Institute of Technology: Pasadena, California)

Hunter, T. R., Cox, P., Benford, D. J., \& Roelfsema, P. R. 2000, AJ, 119,2711

Ivezić, Z̆., \& Elitzur, M. 1997, MNRAS, 287, 799

Ivezić, Z̆., Nenkova, M., \& Elitzur, M. 1997, User Manual for DUSTY, Internal Report (University of Kentucky)

Jørgensen, J. K., Schöier, F. L., \& van Dishoeck, E. F. 2002, A\&A, 389, 908

Mueller, K. E., Shirley, Y. L., Evans, N. J., \& Jacobson, H. R. 2002, ApJS, 143, 469

Kaper, L., Bik, A., Hanson, M. M., \& Comerón, F. 2002, in Hot Star workshop III: The earliest stages of massive star birth, ed. P. A Crowther, ASP Conf. Ser., 267, 95

Kuchar, T. A., \& Bania, T. M. 1994, ApJ, 436, 117

Olmi, L., \& Cesaroni, R. 1999, A\&A, 352, 266

Ossenkopf, V., \& Henning, Th. 1994, A\&A, 291, 943

Plume, R., Jaffe, D. T., \& Evans, N. J. 1992, ApJS, 78, 505

Plume, R., Jaffe, D. T., Evans, N. J., Martin-Pintado, J., \& Gomez-Gonzalez, J. 1997, ApJ, 476, 730

Pickett, H. M., Poynter, R. L., Cohen, E. A., et al. 1998, J. Quant. Spectrosc. Radiat. Transfer, 60, 883

Pollack, J. B., Hollenbach, D., Beckwith, S., et al. 1994, ApJ, 421, 615

Pratap, P., Megeath, S. T., \& Bergin, E. A. 1999, ApJ, 517, 799

Rodgers, S. D., \& Charnley, S. B. 2003, ApJ, 585, 355

Shirley, Y. L., Evans, N. J., \& Rawlings, J. M. C. 2002, ApJ, 575, 337

Thompson, M., Hatchell, J., Macdonald, G., \& Millar, T. 2002, in Hot Star Workshop III: The Earliest Stages of Massive Star Birth, ed. P. A. Crowther, ASP Conf. Ser., 267, 429

Turner, B. E., Chan, K., Green, S., \& Lubowich, D. A. 1992, ApJ, 399, 114

van der Tak, F. F. S., van Dishoeck, E. F., Evans, N. J., Bakker, E. J., \& Blake, G. A. 1999, ApJ, 522, 991

van der Tak, F. F. S., van Dishoeck, E. F., Evans, N. J., \& Blake, G. A. 2000, ApJ, 537, 283

Watson, A. M., \& Hanson, M. M. 1997, ApJ, 490, L165

Wilner, D. J., Ho, P. T. P., \& Zhang, Q. 1996, ApJ, 462, 339

Wyrowski, F., Schilke, P., \& Walmsley, C. M. 1999, A\&A, 341, 882

Wyrowski, F., Gibb, A. G., \& Mundy, L. G. 2002, in Hot Star Workshop III: The Earliest Stages of Massive Star Birth, ed. P. A. Crowther, ASP Conf. Ser., 267, 429

Zijlstra, A. A., Pottasch, S. R., Engels, D., et al. 1990, MNRAS, 246, 217 